\title{
TWO SUTURING TECHNIQUES FOR CORNEAL GRAFTS*
}

\author{
BY
}

\author{
C. R. KANAGASUNDARAM \\ Birmingham and Midland Eye Hospital
}

MANY authorities advocate the use of direct sutures for the fixation of corneal grafts. One argument against their use is the trauma caused by the forceps which are used to support the graft while the sutures are inserted.

Such trauma is not limited to the punctures caused by the teeth of the forceps and the crushing effect of the jaws; lateral tearing of the graft can occur when the graft is supported at one point and the needle inserted to one side of this point. This type of trauma can be avoided by the use of forceps with two pairs of jaws, which support the graft at two points while the needle is inserted into the tissue between them. These double forceps have the disadvantage that they puncture the graft at two points for each suture inserted.

The techniques to be described suggest a means of avoiding damage to lamellar and perforating grafts with forceps while inserting direct sutures.

\section{Lamellar Grafts}

Instruments for counter pressure during the insertion of sutures consist of a ring or twin prongs of metal with a gap in the centre. The tissue to be sutured is supported by the instrument while the needle is passed through the tissue into the gap.

When using this technique for the thin slices of tissue used in lamellar grafting, one finds that the gap in the head of the counter presser is so wide that the corneal tissue dips into it while the needle is being inserted. This prevents accurate and easy placing of the suture. The dipping could be prevented by narrowing the gap in the counter presser but this would increase the chances of damage to the cutting edges of the needle.

Method.-The counter presser used in the technique to be described is made of polythene (Fig. 1, opposite). It avoids the dipping effect mentioned as there is no hole in the head of it. The blade of the instrument supports the tissue from behind while the needle penetrates the graft, and the emerging needle point is made to bite very slightly into the counter presser (Fig. 2, opposite).

The instrument is then withdrawn and is so placed that it supports the back of the graft and the needle point is steadied by lying in the notch at the apex; the needle may then be pushed through the graft sliding all the while in the notch (Fig. 3, opposite). The suture can then be inserted into the recipient bed (Fig. 4, opposite).

Four to six sutures can be inserted into a lamellar graft in this way without once having to touch the donor or recipient with forceps.

* Received for publication August 18, 1960. 


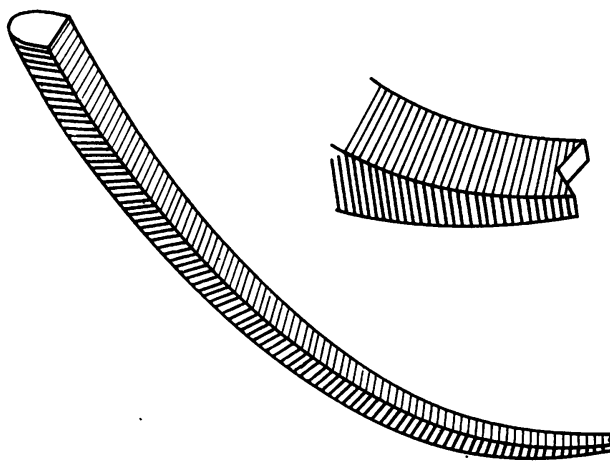

FIG. 1.-Polythene counter presser (actual size). Inset: enlarged view of notch to support needle.

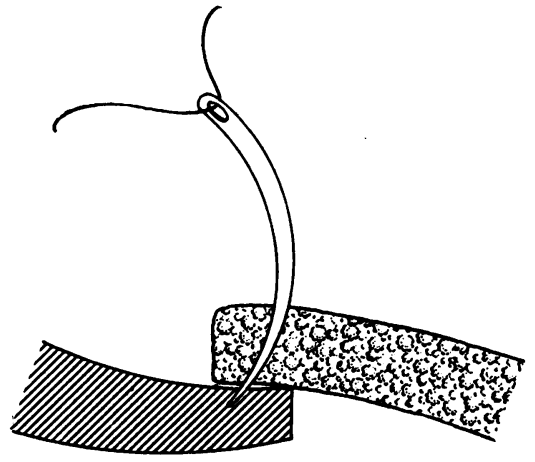

FIG. 2.-The needle is passed through the graft and the point bites into the counter presser.

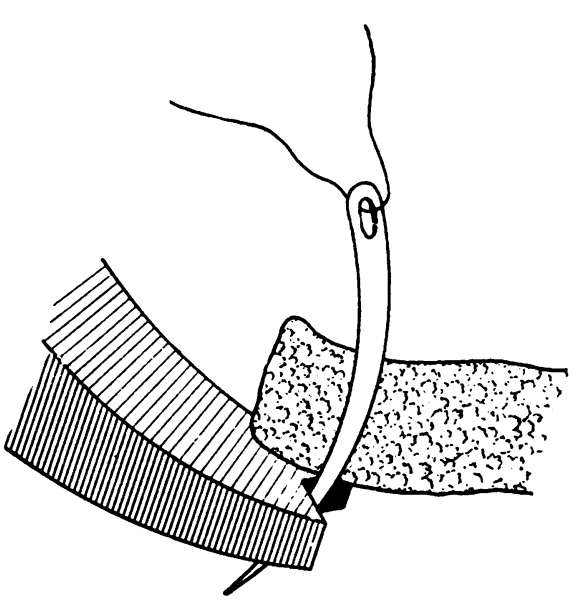

FIG. 3.-The needle is pushed further through the graft, the point being steadied in the notch at the end of the counter presser.

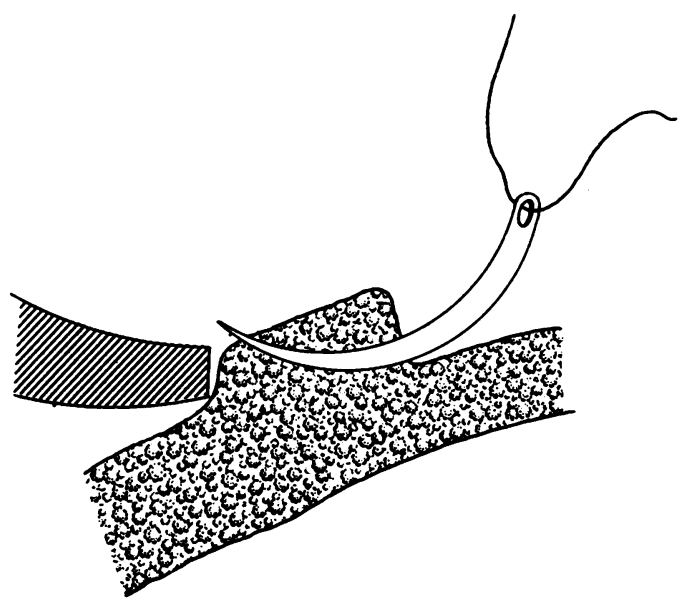

FIG. 4.--Insertion of suture through the lip of the recipient bed, the needle point being guided by the counter-presser.

\section{Penetrating Grafts}

Direct sutures are usually inserted into penetrating grafts after they have been detached and while they are supported by forceps. Several suturing methods which minimize trauma to a graft are being used at present.

Arruga's graft holder enables one to insert sutures into a graft after it has been detached without damaging it with toothed forceps.

The procedure of Teng and Katzin (1952) also provides for the insertion of sutures into the detached graft with little added trauma.

Fixation trauma to the graft can be minimized by tunnelling before completing the section with a thicker thread than that to be used for the final suture (Bietti, 1949). 
Advantages. - The technique to be described has the following points in its favour:

Four to six pre-placed sutures can be inserted into a graft before it is detached from the donor eye.

The whole thickness of the graft is cut through with the same trephine.

The depth of the sutures can be adjusted by the depth of the initial trephine cut.

Forceps are not used on the graft at any stage of the proceedings.

Double-armed filament silk sutures are prepared with the ends fixed to the needles with a half hitch and the free limbs of the sutures cut fairly short. This prevents the tangling of long loose ends.

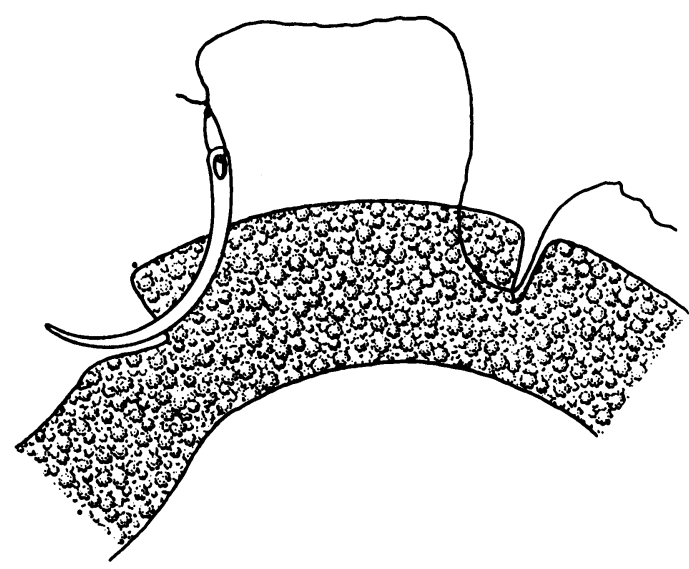

FIG. 5.-Insertion of a suture into a penetrating graft after the initial trephine cut.

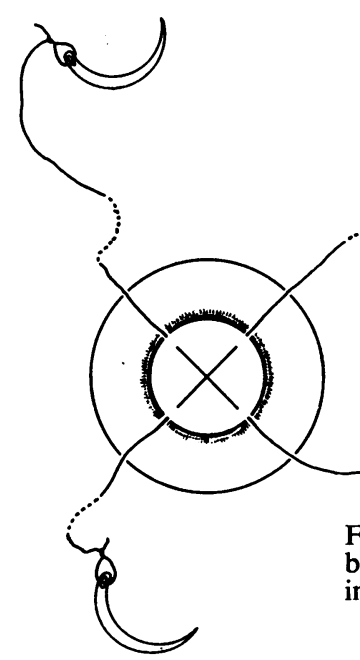

Method.-A trephine cut is made half way into the donor cornea with an instrument which has a detachable blade such as the Franceschetti trephine made by Grieshaber or Down Bros. The doublearmed sutures are then inserted into the graft and brought out through the trephine cut (Fig. 5).

The trephine blade is then removed and the ends of the sutures are threaded through it. This procedure is facilitated by inserting a small-bore polythene tube through the trephine blade, fixing the needle in the lumen of the tube, and so drawing the sutures through the trephine (Fig. 6).

FIG. 6.-Sutures being threaded through the trephine blade with the help of a small-bore polythene tube, after insertion into the partly detached penetrating graft. 
The trephine blade is now slipped down again along the sutures, which are held under light tension, into the original trephine cut (Fig. 7). The light tension on the sutures compresses the inner edge of the graft sufficiently to enable the trephine to slip past the sutures into the bottom of the cut and beyond it without damaging them, but is insufficient to cause any separation of the corneal lamellae. The tension on the sutures is then relaxed and the graft is cut in the usual way using the trephine blade only. Forceps have to be used to fix the recipient cornea while the graft is being sutured to it. Six sutures can be preplaced without becoming tangled. Four sutures at an angle of $90^{\circ}$ or $60^{\circ}$ to each other would make things simpler, additional sutures being inserted in the usual way after the graft is partly fixed to its bed.

When the sutures are threaded through the trephine blade, those nearest the trephine should be passed through first. This allows the strands to lie evenly and enables easy separation of the sutures after the graft is detached.

It is important to hold the trephine blade at right angles to the donor cornea during the cutting of the graft. Otherwise uneven penetration of the donor cornea may result in an early loss of the anterior chamber before the graft is completely detached, and

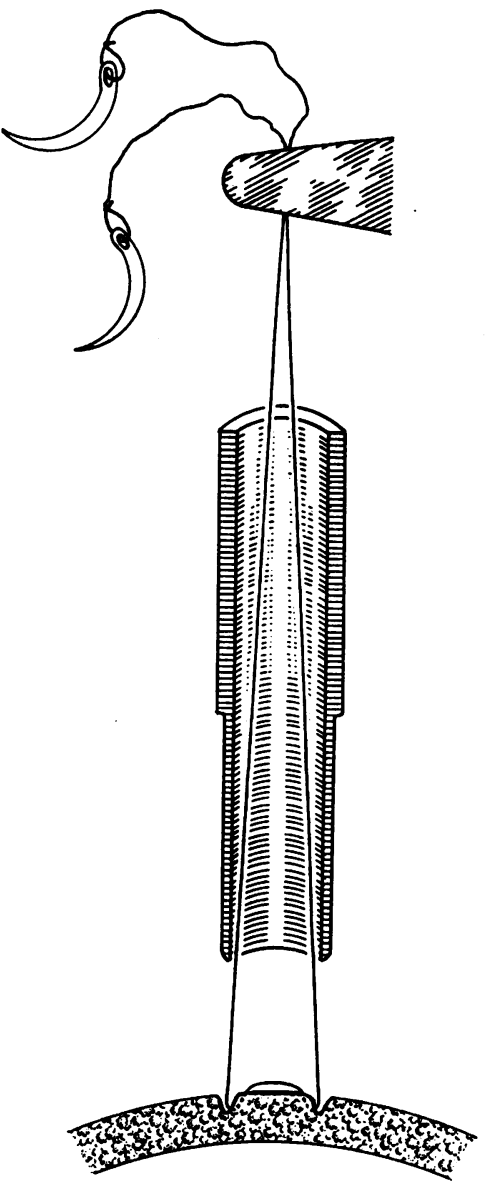

FIG. 7.-Sutures in a partly detached graft being held with forceps under light tension while the trephine blade is slipped back into the cut.

this will necessitate much dissection with corneal scissors or with a knife.

\section{Summary}

Damage to corneal grafts caused by fixation forceps during the insertion of direct sutures is discussed.

Two methods of inserting direct sutures for lamellar and penetrating grafts without the use of fixation forceps are described.

\section{REFERENCES}

ARrugA, H. (1952). “Ocular Surgery”, trans. from 3rd ed. by M. J. Hogan and L. E. Chaparro, p. 375. McGraw-Hill, New York.

BietTI, G. B. (1949). Trans. ophthal. Soc. U.K., 69, 71.

TenG, C. C., and Katzin, H. M. (1952). Amer. J. Ophthal., 35, 587. 